\title{
A Simplified Field-of-View Calibration Technique for an Operating Microscope
}

\author{
A. Hartov, H. Sun, D.W. Roberts, K.D. Paulsen \\ Dartmouth College, Hanover, NH 03755
}

\begin{abstract}
We present a simplified camera calibration algorithm taking advantage of the geometry of our operating microscope, and requiring no special calibration target. Simulations showed that this algorithm is moderately sensitive to inaccuracies in $\mathrm{S}$ and $\mathrm{f}$. For nominal values of $\mathrm{S}_{\mathrm{n}}=6 \mathrm{pix} / \mathrm{mm}$ and $\mathrm{fn}=300 \mathrm{~mm}$, we found that a $5 \%$ deviation in the value of $\mathrm{S}$ results in a mean error of $0.99 \mathrm{~mm}$, while a $5 \%$ deviation in the actual value of $\mathrm{f}$ results in a $0.81 \mathrm{~mm}$ mean error.
\end{abstract}

\section{Introduction}

Our goal is to use the operating microscope's field of view (FOV) as a means to track shift between a preoperative MR and the operating field. This will provide data to update the preoperative study and compensate for the shift [1]. A necessary step consists of being able to predict where a point in world coordinates will appear in the FOV. Determining the required transformation matrices is usually referred to as the „camera calibration“ problem. Camera calibration has been investigated by many and recent contributions include: Zhang [2], Devy et al. [3], Luong et al. [4], Stein [5], Hartley [6], Wei et al. [7], Faugeras [8], and Weng et al. [9], Caprile et al. [10]. It is also discussed in a textbook Gonzalez \& Wintz [11] and Tsai [12]. We sought a much simpler approach allowing calibration on the fly during a procedure, and requiring no special calibration setup.

\section{Materials and Methods}

With a readily identifiable focal point, and the ability to physically make it coincide with any desired world feature we devised a method to express its location in world space using a suitable transformation matrix [13]. The result of this procedure is a vector $\mathrm{V}_{\mathrm{f}}$ representing the location of the focal point in tracker coordinates. The series of transformations needed to map a point from world to image coordinates is:

$$
{ }^{I} P={ }^{I} T_{C} \cdot{ }^{C} T_{T} \cdot{ }^{T} T_{W} \cdot{ }^{W} P
$$

where the subscript and superscripts indicate image (I), camera (C), tracker (T) and world (W) coordinate systems. In equation 1, the location of the world-coordinates 
point, ${ }^{\mathrm{w}} \mathrm{P}$, is known; ${ }^{\mathrm{T}} \mathrm{T}_{\mathrm{W}}$, the world to tracker transformation, is provided by the $3 \mathrm{D}$ positioning system; ${ }^{C} \mathrm{~T}_{\mathrm{T}}$, the tracker to camera transformation, needs to be computed and the camera to image transformation, ${ }^{\mathrm{I}} \mathrm{T}_{\mathrm{C}}$, is determined as explained here.

In the following discussion the desired point is known in tracker coordinates (i.e. the right-most multiplication in (1) has been performed). We expand here equation 1 in matrix form:

$$
\left[\begin{array}{c}
c \cdot x_{I} \\
c \cdot y_{I} \\
c
\end{array}\right]=\left[\begin{array}{cccc}
S_{x} & 0 & 0 & 0 \\
0 & S_{y} & 0 & 0 \\
0 & 0 & 1 / f & 0
\end{array}\right] \cdot\left[\begin{array}{cccc}
r_{1} & r_{2} & r_{3} & t_{x} \\
r_{4} & r_{5} & r_{6} & t_{y} \\
r_{7} & r_{8} & r_{9} & t_{z} \\
0 & 0 & 0 & 1
\end{array}\right] \cdot\left[\begin{array}{c}
x_{T} \\
y_{T} \\
z_{T} \\
1
\end{array}\right]
$$

From (2), it can be seen that $\mathrm{c}=\mathrm{z}_{\mathrm{C}} / \mathrm{f}$, where $\mathrm{z}_{\mathrm{C}}$ is the $\mathrm{z}$ coordinate of the point in camera coordinates. This coefficient is the nonlinear component of the overall transformation process, since it includes the $\mathrm{z}_{\mathrm{C}}$ term and since the homogenous image coordinates must be normalized, (divided by c) to obtain $\mathrm{x}_{\mathrm{I}}$ and $\mathrm{y}_{\mathrm{I}}$. Sx and Sy are the scaling factors translating distance $(\mathrm{mm})$ to pixels in our system $\mathrm{Sx}=\mathrm{Sy}$ (square pixels). The term $\mathrm{f}$ is the focal length of the optical system; when the focal point is aimed at the point of interest, $\mathrm{z}_{\mathrm{C}}=\mathrm{f}$ and $\mathrm{c}=1$. Because of the nonlinear term in the camera to image transformation ${ }^{\mathrm{I}} \mathrm{T}_{\mathrm{C}}$ it is not possible to compute it directly. We chose to determine $\mathrm{Sx}$ and Sy directly from images and we also assume $f$ to be the focal length specified by the microscope's manufacturer. The matrix containing the r1...r9 and tx...tz elements $\left({ }^{\mathrm{T}} \mathrm{T}_{\mathrm{C}}\right)$ relates tracker and camera spaces, and is the only term that needs computing. Having obtained estimates of the components of ${ }^{\mathrm{I}} \mathrm{T}_{\mathrm{C}}$, we are able to solve for the remaining unknown terms using points whose coordinates are known in tracker and image frames.

\section{Results}

\subsection{FOV Calibration: Simulations Results}

Sx and Sy were fond from images of a ruler in the focal plane at each magnification setting. Evaluation of the FOV calibration is based on computer simulations at 4 different magnification settings with nominal values for $\mathrm{Sn}=6,12,24$ and $48 \mathrm{pix} / \mathrm{mm}$, (zoom factors of 1, 2, 4 and 8) and a fixed nominal value of $300 \mathrm{~mm}$ for the focal length fn. We used a 9x9 grid of test points forming a $20 \mathrm{~mm}$ square in camera space. From the test points defined in camera coordinates, homologous sets were produced in tracker, world and image coordinates. The image coordinates were computed using an actual focal length fa and an actual scaling factor Sa. These were varied by $\pm 10 \%$ from their nominal values fn and $\mathrm{Sn}$ in 21 increments. Errors were computed from the Euclidean distances between the correct and calibrated points are summarized in table 1. 


\section{Tables}

Table 1. Error in image point projection due to a disparity between the nominal $\left(\mathrm{S}_{\mathrm{n}}\right.$, $f_{n}$ ) values for the scaling factor and focal distance and their actual values.

\begin{tabular}{|c|c|c|c|c|c|c|c|c|}
\hline \multirow{3}{*}{$\begin{array}{l}\text { Conditions } \\
\text { All units mm }\end{array}$} & \multicolumn{4}{|c|}{$10 \%$ error } & \multicolumn{4}{|c|}{ error } \\
\hline & \multicolumn{2}{|l|}{$\mathrm{S}$} & \multicolumn{2}{|l|}{$\mathrm{f}$} & \multicolumn{2}{|l|}{$\mathrm{S}$} & \multicolumn{2}{|l|}{$\mathrm{f}$} \\
\hline & mean & $\max$ & mean & $\max$ & mean & $\max$ & mean & $\max$ \\
\hline $\mathrm{Sn}=6, \mathrm{fn}=300$ & 1.715 & 2.8808 & 1.8865 & 3.0641 & 0.9928 & 1.7552 & 0.8097 & 1.4142 \\
\hline $\mathrm{Sn}=12, \mathrm{fn}=300$ & 1.7175 & 2.8808 & 1.6824 & 2.7735 & 1.0373 & 1.7552 & 0.8244 & 1.3176 \\
\hline $\mathrm{Sn}=24 \mathrm{fn}=300$ & 0.9545 & 1.4404 & 0.8591 & 1.2964 & 0.5816 & 0.8776 & 0.4407 & 0.6482 \\
\hline $\mathrm{Sn}=48 \mathrm{fn}=300$ & 0.5464 & 0.7202 & 0.4918 & 0.6482 & 0.3329 & 0.4388 & 0.2459 & 0.3241 \\
\hline
\end{tabular}

\section{References}

1. Roberts, D. W.; Miga, M. I.; Hartov, A.; Eisner, S.; Lemery, J. M.; Kennedy, F. E.; Paulsen, K. D. „Intraoperatively Updated Neuroimaging Using Brain Modeling and Sparse Data.“ Neurosurgery, Vol. 45, No. 5, Nov. 1999.

2. Zhang, Z. „A Flexible New Technique for Camera Calibration“ Technical Report MSR-TR90-71. Microsoft Research. http://research.microsoft.com/ zhang

3. Devy, M; Garric, V.; Orteu, J. „Camera Calibration from Multiple Views of a 2D Object, Using a Global Non Linear Minimization Method.“ Proc. IROS 97, pp 1583-1589.

4. Luong, Q.-T.; Faugeras, O.; „Self-Calibration of a Moving Camera from Point Correspondences and Fundamental Matrices. Int. J. of Computer Vision. 22(3):261-289, 1997.

5. Stein, G. „Accurate Internal Camera Calibration Using Rotation, with Analysis of Sources of Error.“ Proc. 5th Int. Conf. On Computer Vision. Pp 230-236, 1995.

6. Hartley, R. I. „An Algorithm for Self Calibration from Several Views.“ Proc. IEEE Conf. On Computer Vision \& Pattern Recognition. Pp. 908-912, Seattle, WA, 1994.

7. Wei, G.; Ma, S. „Implicit and Explicit Camera Calibration: Theory and Experiments.“ IEEE Trans. On Pattern Analysis and Machine Intelligence. 16(5):469-480, 1994.

8. Faugeras, O.; „Three-Dimensional Computer Vision: a Geometric Viewpoint.“ MIT press, 1993

9. Weng, J.; Cohen, P.; Herniou, M. „Camera Calibration with Distortion Models and Accuracy Evaluation.“ IEEE Trans. On Pattern Analysis \& Machine Intelligence, 14(10):965980. 1992.

10. Caprile, B.; Torre, B. „Using Vanishing Points for Camera Calibration.“ Int. J. of Computer Vision, 4, pp. 127-140, 1990

11. Gonzalez, R. C.; Wintz, P.; „Digital Image Processing.“ 2nd edition. 1987.

12. Tsai, R. Y. „A versatile camera calibration technique for high-accuracy $3 \mathrm{D}$ machine vision metrology using off-the-shelf TV cameras and lenses." IEEE Journal of Robotics and Automation, Vol. RA-3, No. 4, Aug. 1987.

13. Hartov, A.; Eisner, S. D.; Roberts, D. W.; Paulsen, K. D.; Platenik, L. A.; Miga, M. I. „Error analysis for a free-hand three-dimensional ultrasound system for neuronavigation.“ Neurosurgical Focus Vol 6, No. 3, article 5, 1999. Online publication: http://www.neurosurgery.org/focus/mar99/6-3-5.html 